This is the peer reviewed version of the following article: Yule, J. A., and Tinson, J. S. (2017) Youth and the sociability of "Vaping". J. Consumer Behav., 16: 3-14, which has been published in final form at https://doi.org/10.1002/cb.1597. This article may be used for non-commercial purposes in accordance With Wiley Terms and Conditions for self-archiving. 


\title{
Youth and the Sociability of 'Vaping'
}

\author{
Jennifer A Yule ${ }^{1}$, Julie S Tinson*2
}

${ }^{1}$ Northeastern University Boston, 360 Huntington Ave,

Boston MA 02115.

Email: je.yule@neu.edu

Phone: +1 617-373-2812

Fax: +1 6173738366

*Corresponding Author

${ }^{2}$ Stirling Management School, University of Stirling, Scotland, UK, FK9 4LA

Email: j.s.tinson@stir.ac.uk

Phone: +44 (0)1786 467389

Fax: +44 (0)1786 467400

\section{Author biographies}

Jennifer Yule is an Academic Specialist in Marketing at Northeastern University, Boston, MA. Her research is centered around consumer well-being and is focused on two specific areas. First, the healthcare industry where current projects focus on the patient experience in hospital. Second, policy related projects which currently focus on e-cigarette consumption.

Julie Tinson is Professor of Marketing at the University of Stirling where she principally teaches Marketing Management. Her research interests are motivated by an interest in the relative impact of transition on consumption and identity formation using ritual practice as a context. Publications have centered on the inter-relationships between adolescent consumer behavior and the social factors that affect the consolidation of identity positions.

\section{Youth and the Sociability of 'Vaping'}

\section{Abstract}


This article examines the contemporary and evolving social ritual of vaping (also known as the use and consumption of e-cigarettes). This novel research finds a typology of users in a heterogeneous youth market and shows how the introduction of this new practice challenges existing ritual boundaries. Previous research has focused on the de-marketing of tobacco and smoking cessation. Here, Virtuous, Dynamic, Vulnerable and Invisible Vapers are identified with each demonstrating differing levels of emotional engagement and characteristics of vaping behaviors. Utilizing Collins' theory of interaction rituals as a lens, this qualitative study explores the sociability of vaping practices to gain a deeper understanding of the enduring appeal of vaping and the subsequent consequences for youth 'smoking' behaviors. While some vapers advocate the health benefits of this relatively new practice, the use of ecigarettes is also used as a form of resistance as well as mechanism for misbehavior.

\section{Key words:}

E-cigarettes, interaction ritual, health, symbolism, sociability 


\section{Youth and the Sociability of 'Vaping'}

Rituals represent boundaries of inclusion and exclusion that are, "at times contested by persons located in various relationships to those boundaries" (Collins, 2004: 297). Rituals are "highly varied types of expressive behavior that occur in quite diverse settings" (Rook, 1985, pg. 252) and include tobacco consumption, which has been subject to well documented social control (Choi and Forster, 2013), 'de-marketing' (Shiu et al., 2009) and health regulation (Marlow, 2014). A relatively new addition to the marketplace and related social rituals, the e-cigarette, has been met with a mixed response. Proponents note the success of ecigarettes at reducing tobacco consumption (Brown et al., 2014; Cahn and Siegel, 2011) while adversaries seek to understand and identify the risks of these chemically based and branded products (Bialous and Sarma, 2013; Etter, 2010; Etter and Bullen, 2011). Of particular concern are recent figures which suggest that adolescent use of e-cigarettes has surpassed the use of tobacco cigarettes (Centers for Disease Control and Prevention [CDC], 2015 ) with health officials apprehensive of a 'gateway effect' for youth cigarette smoking after years of decline in cigarette use by minors (Paradise, 2014). This raises the following questions: What facets of this enduring social ritual particularly appeal to the youth market and how do new products shift ritual boundaries (e.g. alter consumer behavior) and subsequent opposition to such practices?

E-cigarettes were initially designed to deliver a safer alternative to nicotine consumption than tobacco products (Cahn and Siegel, 2011) and, as a consequence of the initially unregulated nature of the market there are a plethora of devices on offer (Marlow, 2014). While there is variation in product design across brands, most e-cigarettes contain a battery triggering a heating action when users inhale. This heat then connects with a solution, usually containing nicotine, which transforms into vapor (Brown et al., 2014). The term 'vaping' is used by advocates of e-cigarettes as they do not smoke but rather inhale vapor (Borland, 2011). The user is able to both customize vaping equipment and liquid to suit their preferred taste. This is reflective of the development of playfulness in the vaping market where deconstructing and reassembling products or brands is manifest in developing social links (Cova et al., 2007; Goulding et al., 2013). As the value of the $\$ 2 \mathrm{~b}$ e-cigarette market continues to grow (Centers for Disease Control and Prevention [CDC], 2013; Euromonitor, 2012), understanding the more subtle behaviors associated with this 'vaping' ritual will offer insight into the notion of sociability in this context. 
To extend prior research, we conducted a study with 'vapers'; namely those who practice, deliberate, create and introduce vaping behaviors. The key contribution of this study is a typology of vaping behavior that offers insight and understanding into this heterogeneous market. This exploratory study was designed to understand the fluidity of the vaping ritual parameters with a view to investigating our key research questions. Firstly, how do youth explain their engagement with vaping? And subsequently how do these rationales reflect and develop the established tobacco rituals of relaxation and withdrawal, carousing and elegance (Collins, 2004)? We also explore the creation of new social boundaries namely, where the ingroup ends and the out-group begins, and social identities e.g. individual affiliation with own group/s as a consequence of this ritual practice (Cova et al., 2007). This leads to our second research question. How do those less familiar with the ritual practice of vaping and the subtle intricacies of shared knowledge learn about what is being consumed; accessing communal experiences and related membership of such collectives? This study also affords an opportunity to explore sociability in this context which generates particular types of emotional energies in groups (Collins, 2004). This leads to our final research question which concerns the collective nature of vaping. If this practice is inherently social, in what ways can any opposition to such practices be effective? Collins' (2004) theory of interaction ritual chains is employed here to understand the meanings, interpretations and boundaries associated with vaping.

The rituals associated with tobacco use provide a long history on which to build our research in this contemporary context. Many forms of tobacco have been used in a variety of social group settings with different configurations of tobacco becoming more or less fashionable across cultures as well as changing over time (Gilman and Zhou, 2004; Wearing and Wearing, 2000). We provide a typology of vapers to illustrate types of attitudes and behaviors associated with this practice and move beyond previous studies associated with tobacco consumption to contribute to an understanding of the social nature of vaping. The following section provides an overview of the concepts relating to rituals and the interrelationship between these and the development of social behaviors. This provides a basis on which to develop our understanding of vaping not as an individual or disparate practice but rather as one which encourages collective interpretation, playfulness and the creation of new social identities as well as shifting ritual boundaries. 


\section{Interaction rituals}

While there has been an array of studies in marketing that have explored the historical context, symbolic significance and commercialization of ritual events, these have typically focused on high as opposed to low intensity rituals e.g. weddings, death, birth and divorce [see for examples: Bonsu, 2001; McAlexander, 1991; Otnes and Lowrey, 1993; Ozanne, 1992]. Low intensity rituals are characterized as activities that involve a varying degree of formality and process and there are three main low intensity tobacco rituals which offer insight for exploring vaping practices (Collins, 2004). First, relaxation or withdrawal rituals are characterized by removal from day-to-day anxieties. The pressures and excitement associated with work and social burdens can lead to a desire to escape. Secondly, carousing or enjoyment rituals are typically associated with riotous action and alcohol consumption. This reinforces the extent to which tobacco use has been viewed as an 'acceptable' form of rebellion (Hendlin et al., 2010). Finally elegance rituals "convey an aesthetic impression of the actor as a categorical identity within the status hierarchy" (Collins, 2004: 306) illustrating how smoking has historically been used as a way in which to denote situational stratification or social hierarchy.

Building on the work of Durkheim (1964) and Goffman (1967), Collins (2004) further examines the notion of tobacco consumption in the context of interaction ritual chains. That is, rather than considering high or low intensity rituals as unique or disparate events, Collins (2004) posits that each ritual event will generate positive or negative emotional energy that will then impact on the motivation of individuals to engage in both the original ritual and new future rituals (see Figure 1). The interaction ritual chain consists of ingredients, processes and outcomes and there will be different levels of individual and collective engagement and emotional energy generated by each ritual occurrence. The ingredients of a ritual are: an object or activity, a shared mood, barriers to outsiders and a focus of attention. It is important to recognize in the context of vaping that this ritual can be an inclusive or exclusive as barriers to outsiders would include lack of knowledge to access this type of activity.

Similarly the processes associated with tobacco consumption create solidarity within the group and such practices can lessen hierarchical barriers between individuals (Brown, 2011). Belk et al. (1989) note that a sense of community can be nurtured where collective values are cultivated. These processes, which are often transitory, lead to outcomes that include standards of morality as well as symbols (Collins, 2004). "The increased levels of EE that results from entrainment encourage individuals to seek out similar rituals" (Brown, 2011: 124). As confidence, enthusiasm, initiative and pride can be the long-term outcomes from 
engaging in such social practices (Collins, 2004), exploring the sociability of vaping through the lens of interaction ritual chains offers an explanation of how and why this enduring social ritual appeals to the youth market.

[Insert Figure 1 about here]

\section{Sociability and interaction}

The introduction of vaping lounges and vape shops with social spaces to facilitate communal experiences of consumption has presented a variety of opportunities to develop vaping groups and enhance sociability. Sociability is the relative disposition to engage with others where "associations are accompanied by a feeling for, by a satisfaction in, the very fact that one is associated with others and that the solitariness of the individual is resolved into togetherness, a union with others" (Simmel and Hughes, 1949: 255). Accepting that vaping as a low intensity ritual can facilitate and/or enhance sociability we anticipate that 'vapers' are likely to be tribal as opposed to being part of a sub-culture or brand community as they will typically enjoy membership of a variety of groups (as opposed to one dominant hierarchical collective). While they may exhibit brand preference, the emphasis is expected to be on the shared experience vaping offers (Cova and Cova, 2002). Interestingly the characteristics of tribes are reflected in the observations of Simmel and Hughes (1949: 255) on sociability, "since in sociability the concrete motives bound up with life-goals fall away" and consequently "the free-playing, interacting interdependence of individuals ...operate with so much the greater effect".

Examining sociability in the context of vaping will generate insights as this is a relatively new phenomenon. However, it has long been established that tobacco consumption and the practices therein lead to enhanced sociability (Room, 2004; West et al., 1984). Importantly sociability is a major component of extraversion (Gilbert, 1995) which is correlated with smoking in adults. While higher levels of sociability have been linked to greater use of tobacco, other substances (Chassin et al, 1993) and subsequent 'misbehavior' (Gerrard et al, 1996) propensity for sociability (or being an extravert) does not mean there is a greater susceptibility to smoking (Hampson, 2007). Engagement with and commitment to vaping will vary and evolve but how this manifests in relation to sociability under-researched. Additionally the outcomes associated with vaping individually or collectively have not been fully explored although they are pivotal to an understanding of how and why youth engage with such enduring social rituals. Further, given the low intensity and contemporary nature of 
vaping, understanding how these practices facilitate the production or customization of market offerings will also generate insight into youth consumer behaviors. This, in turn, will afford academics, marketers and policy makers an understanding of the diversity of practice as relates to youth consumer vaping behaviors.

Importantly, this study is the first to examine vaping from the perspective of social and ritual behavior offering contextual insights and theoretical contributions. Specifically this research contributes to extant studies by offering the first qualitative typology of vaping behavior illustrating the nature and complexity of this market and how it compares to and differs from tobacco smoking ritual behavior. Through the lens of interaction ritual chains (Collins, 2004) we uncover that barriers to access, customization and knowledge transfer are key features of this activity. We also identify a group of vapers who are resistant to the vaping community. These individuals lack engagement with other vapers and could be considered strangers (Gronnow, 2004) who wish to dis-identify with the norms and behaviors associated with the vaping group/s. Finally we highlight the popular use of vaping as a covert method of marijuana consumption which has implications for our understanding and management of consumer misbehavior (Fullerton and Punj, 1993).

\section{Method}

Adopting an interpretivist perspective, a total of 20 in-depth interviews were conducted with males and females in Massachusetts, USA (see Table 1). This is a smoke-free state and was specifically chosen because of the restrictions on vaping and what data may reveal about how the protection offered by the state law is circumvented. After a process of informed consent (Miles and Huberman, 1994), respondents were invited to take part. The research was conducted over a one month period in summer where limitations on vaping in enclosed spaces would be less apparent; with individual interviews providing an opportunity to explore a priori and emergent themes in relation to vaping practices. As the 'vapers' had varying degrees of experience and used e-cigarettes differentially, access to and the fluidity of vaping parameters and practices were comprehensively examined.

As health officials are concerned that vaping will create a 'gateway effect' for youth cigarette smoking after years of decline in cigarette use by minors, a youth sample was chosen for this study in part to examine the likelihood of such a prediction. Consequently a purposive sampling approach identified those in 'emerging adulthood'. The idea behind this concept is that, "emerging adulthood is a distinct period demographically, subjectively, and in terms of identity explorations" (Arnett, 2000, Pg. 469). As such it is recognized as a 
contemporary way in which to conceptualize (and research) those in their later teens to their mid-to late 20s in industrialized societies. Additionally as this youth sample are likely to be future and/or long-term users of vaping products, their insights are useful for marketers and policy makers. A semi-structured interview guide was developed for the data collection (see Appendix 1). The questions were designed to explore any rituals associated with vaping, to contextualize the experience of vaping, to understand the level of engagement relative to their own social groups, to encourage narratives of consumer playfulness and to probe for discernible resistance to the protection offered by state law. The interview data collection was sufficient to ensure saturation (Guest et al., 2006).

For the analysis of the interviews, an interpretive analytic stance was adopted drawing on the transcriptions. The analysis of the data explored themes in the responses of 'vapers' using the methods outlined by Strauss and Corbin (1990). Each interview was examined to gain a holistic understanding of the respondent following Thompson and Hirschman (1995); noting themes in the margin as they emerged. This coding was driven deductively by the original issues identified in the literature review and inductively by searching for emergent themes. All the themes were reviewed through iterations of comparison and re-reading. The interpretations developed were as a consequence of the relationship between emerging insights and prior assumptions (Spiggle, 1994). The authors brought different perspectives to the analysis and interpretation. Both were involved in the design of the interviews and each independently looked for commonality in interpretation through discussion.

\section{Findings}

The interviewees demonstrate that vaping is used differentially; both as a healthier form of smoking behavior than tobacco as well as for 'playfulness' or social purposes. Participants offered a variety of ways in which they engage in vaping practices including customization, information seeking and knowledge transfer. With the exception of the social benefits proffered by respondents, this study extends previous research which suggests smoking-type behaviors relate to escape, rebellion and identity positioning (Collins, 2004). While all vapers advocate the use of e-cigarettes, importantly there are significant differences between the motivations vapers offer for engaging in such practices. For example, former tobacco smokers prefer vaping as they perceive their breathing is less affected by vaping than when they were smoking. For non-tobacco smokers vaping is considered as a 'healthy' alternative to tobacco smoking not only because there is no offensive smell but as a consequence of the glycerin based liquid used in the e-cigarette. This is perceived as a natural 
product and therefore not harmful. Furthermore, in the segments identified here there are clear distinctions between those vaping to engage in social behaviors and those vaping to simply improve their health outcomes.

In the findings section we present four consumer segments of vapers and reflect the key characteristics that differentiate each consumer type. For each grouping we explore a number of salient issues, including (i) the role of vaping as it pertains to relaxing, carousing and social stratification, (ii) individual and collective participation associated with vaping as well as related knowledge, vaping practices and ritual interaction outcomes and finally, (iii) any enduring aspects of this social ritual that appeal to the youth market. These topics are examined by using a typology to enable an incisive understanding of the data (see Figure 2). Typologies are designed to stimulate thinking (Mills and Margulies, 1980) and afford the opportunity to examine multifaceted experiences in a single construct (Blau and Scott, 1962). This typology is not designed to 'freeze meaning' (Slater, 1997); rather it is illustrative of types of groups that vaping consumers can belong. The four types of e-cigarette consumers depicted here are Virtuous Vapers, Dynamic Vapers, Vulnerable Vapers and Invisible Vapers. Within the typology there is a divergence between social engagement and health benefits which will be examined in relation to this low intensity ritual and associated outcomes.

[Insert Figure 2 about here]

\section{Virtuous Vapers}

Virtuous Vapers are typically characterized as former tobacco smokers who view vaping as a healthy way to facilitate their smoking cessation. These vapers focused on the functional attributes of the devices and enjoy the perceived cost benefits when compared to tobacco smoking. Their behaviors associated with vaping are less playful or creative than those of other vapers and the perceived health benefits of vaping are of far greater importance than the potential social experience. Andy is typical of virtuous vapers in describing his attitude toward vaping as a tobacco replacement device:

I mean, it started as a nicotine delivery alternative, and that's pretty much what it is still. I mean, I guess you could say it's a hobby, because I do a lot more, you know, hands-on stuff with it now. But it's not really... a social thing for me. 
The practical motivations driving these participants mean that information seeking related to health benefits in this group is enhanced comparatively with other segments. This is reflected in a desire for information about the mechanics of the devices as well as evaluating the risks and health properties associated with vaping. Virtuous Vapers approach vaping as a high involvement decision that requires careful consideration and perform data mining techniques characteristic of a younger demographic (Albritton, 2015); often through social networking and online forums. In particular, studies that consider the potential detrimental effects of vaping are carefully scrutinized as Anthony describes here:

I like to hear what people have to say... I was on a subreddit [a niche discussion forum] and read that people were unhappy with the parameters of the experiment [about the detrimental health effects of vaping]. That was what, you know, enticed me to go and actually look up the study, and figure out why. So that was where I got the idea was from Internet forums...

The inquisitiveness Virtuous Vapers have about the health effects of vaping also extend to the mechanics involved and the optimizing of devices for maximum effect. These participants typically have considerable knowledge of the ritual paraphernalia and focus on the practical attributes of the product as opposed to the symbolic. As Virtuous Vapers use vaping as a cessation aid, the appeal of being able to control the level of nicotine delivery is significant. As Ivan explains, he can customize and develop his own 'rig' to personal specifications and through the use of the word 'ridiculous' is dismissive of those who use vaping to be creative or playful:

...there's a lot of customization that goes on with it. Like, you can build your coils and, depending on how many times you wrap it or what gauge you have, it'll be a different resistance, and that results in, different heat. So if you want something less intense, you build a higher resistance. If you want some ridiculous cloud-chasing thing, you build really low and have a ridiculous amount of airflow, and you get like no flavor.

While knowledge of the ritual paraphernalia and purchase of the 'correct' rig is important to maximize the practical experience for Virtuous Vapers, cost is also identified as a key motivator for this group. Nate and Andy are clear about the savings they have made by stopping cigarette smoking and opting to vape. While Andy emphasizes the cost of cigarettes, Nate focuses on the price savings of e-cigarettes: 
...you know, the amount of money that cigarettes cost is ridiculous. And in the long term, you know, hands down you would save money switching to e-cigarettes, which I did, and, you know, it's easy to calculate how much you would have spent on cigarettes, compared to using an e-cig. (Andy)

...I used to smoke a pack a day. So that's, that's you know six to seven dollars every day, like you know, about five times a week. This one is [his vape], like this is about like, like I spend about thirty dollars every like two weeks on the coals and the liquid and stuff like that. (Nate)

Although cost savings are a driver, the boundaries of this low intensity ritual for Virtuous Vapers are invariably related to health properties and there is evidence here that Virtuous Vapers want to seek information to contain extant boundaries. While there is evidence of customization and innovation as suggested by Cova et al. (2007) this is limited and these participants focus on the functional aspects of this practice to reinforce the health benefits while seeking additional information to support and advocate such behavior. This segment is opposite in their characteristics to Dynamic Vapers who often view the vaping ritual as a performance (Belk et al., 1989) while illustrating playfulness (Cova et al., 2007) and opinion leadership (McCracken, 1986) with specific reference to how and in what ways vaping can facilitate sociability (Simmel and Hughes, 1949).

\section{Dynamic Vapers}

Dynamic Vapers value the perceived social benefits of vaping over the health benefits sought by Virtuous Vapers. They identify as key opinion leaders e.g. those who influence the behavior of others, as they report they are recognized as educating others on vaping practices. This consequently reduces barriers to those interested in becoming part of the vaping community. These participants generate the most positive emotional energy (EE) (Collins, 2004) through vaping relative to other segments and as such show confidence and enthusiasm as a consequence of engaging in this social interaction. Duane illustrates this through exploring his own interaction with a roommate:

She'll smoke a bowl, I'll vape a little bit, and we'll, like, talk about our day, talk about some of those problems, whatever, just chat it out. And then me and her smoke good amounts, so we'll just keep talking. And then suddenly... we'll be like engrossed in a 15-minute conversation! 
The high EE generated through vaping often leads to Dynamic Vapers defending the activity as well as promoting the social benefits of vaping to others. Indeed our Dynamic Vapers express feelings of righteousness about vaping which Kemper (1990) drawing on Durkheim (1954) refers to as moral sentiment. This reflects the strong belief of Dynamic Vapers that they are doing something 'right' or proper. Importantly moral sentiment is perceived through feeling rather than reason (Calhoun, 1980). Here, Randy expresses his positive EE through promoting vaping as an aid to smoking cessation but does not offer factual information in the same way Virtuous Vapers are inclined to do:

Yeah. I think it's a great way to quit, and even if you don't want to quit, it's -- I mean,

I think it is better for you. It's not exactly good for you, but it is better.

Similarly enthusiasm for vaping can translate into opinion leadership behavior which is supported by extensive knowledge of creative practices and advocacy (Kemper, 1990) as Katie explains:

...so my friends who are smokers and are trying to quit, they have some cheap little vapes, which don't produce, like, any type of clouds that mine does. And I'm always like, hey, if you really want to try to help yourself, this might help even more. Then they'll try mine, and they'll be like, "Oh yeah, wow, like, that's totally different."

Here some of the complexity involved in vaping with disposable e-cigarettes is underscored. While the practice would seem to be easily accessible, the range of paraphernalia and knowledge required to vape socially can create barriers to outsiders.

Learning to recognize the symbols and symbolism

Dynamic Vapers are highly knowledgeable and revel in all of the positive social consequences of vaping. They have enhanced social capital (Bourdieu, 1986) relative to other segments; giving them a highly stratified status in the vaping community. This suggests for these participants, rather than vaping simply being a tribal practice as anticipated, there are elements of hierarchy and sub-culture (Cova and Pace, 2006) that go beyond playfulness and creativity. For example, participant Duane is passionate about his advanced and technical 'Pax 2' vaping device and uses it at parties to engage others in conversation. In this environment the detailed discussion of the device is the focus of attention which leads to a shared mood or emotional experience (Collins, 2004). Those in the group are collectively admiring the device as well as the symbolism of the 'rig'. 
Well, in terms of vaping weed, people think that it doesn't get you as high, but [vaping] is very social. I think it's very social, because, like, have this big rig! First of all, when you bring it out, people are like, "Yo, that's so cool! Can you explain it?" So people automatically think you're just, like, a huge pothead because you have this vape already. And so it's cool.

In contrast to Virtuous Vapers, Duane highlights the symbolic importance of his device at parties or social events. This form of conspicuous consumption (Rojek, 2000) is being used to make a symbolic statement about self-identity where a desired image can be crafted and projected to others with potential social and relationship benefits (Leventhal et al., 1991).

Katie describes a similar experience when she is blowing smoke rings at a party; relishing becoming the center of attention as a consequence of this skill. The outcome for Katie is high EE with an increased likelihood of further engagement in in the original or new social interaction/s (Collins, 2004). The social value (Sheth et al., 1991) of vaping is also evident here as is the role that knowledge or skill has in creating an impression (Schlenker, 1980). Katie is using vaping as a means of impression management which is raising her selfesteem and subsequently impacting on ability to control her social environment (O'Callaghan and Doyle, 2001). While high levels of EE facilitate further engagement, the presence of high EE is also a product of the collective experience of vaping with others. As Mike illustrates, the role of others is significant in vaping practices and related expertise:

I mean, when I'm vaping with my friends, we're more likely to do like smoke rings and stuff. But if I'm vaping by myself...I'm not going to be like doing tricks.

As "ritualization is best reconstructed in terms of social practices that which are situated and performed" (Hughes-Freeland and Crain, 1998: 2), it is the Dynamic Vapers segment that facilitate the development and evolution of creative practices associated with this low intensity vaping ritual. These findings also support the notion that without an audience a ritual performance has limited social value (Deighton, 1992). While Vulnerable Vapers are also inclined towards the sociability of vaping, their behaviors tend towards resistance.

\section{Vulnerable Vapers}

Hedonistic and carousing describe the key behaviors associated with Vulnerable Vapers. While they are similar to Dynamic Vapers in that they recognize the social value of 
vaping, they are less likely to be opinion leaders. They are more inclined to heavy use of vaping equipment to aid tobacco cessation or facilitate drug use as well as being pre-disposed to using disposable e-cigarettes. Their behavioral motivation is rooted in social acceptance and group belonging with a strong emphasis on the importance of the social experience associated with this low intensity ritual. This segment are 'followers' as can been seen in the example of Alexa. She described her desire to try to vape because her friends were vaping and she "just thought it looked cool". This motivation translates into low involvement decision making whereby little is known about the health effects and knowledge of the device/s is limited. Common to this group is the purchase of disposable e-cigarettes as Jake discusses:

Yeah, I'd say like almost 100\% of the time, I'm just going to grab a reusable or disposable one and just you know, getting some puffs in.

This purchase behavior is in stark contrast to the Virtuous Vapers who are disparaging of disposable e-cigarettes as it denotes lack of knowledge and appreciation of the complexity of the activity. This tribal linking value (Brown, 2007; Brownlie et al., 2007) that is developed by Vulnerable Vapers is based on a shared experience of vaping together, blowing smoke rings and conversing about a common topic. Tyrone explains the role of his ecigarette in social situations:

...breaking the ice in terms of conversation, you have something that you all have in common. You can talk about your different flavors, the brand. There is a history and a commonality between other people.

In this way sociability is an important factor driving e-cigarette consumption among this group and we see specific carousing rituals evidenced here as vaping is regularly part of partying and alcohol consumption (Collins, 2004). While historically tobacco consumption has been viewed as an acceptable form of rebellion (Hendlin et al., 2010) there is also evidence of vaping being used as a form of consumer misbehavior (Fullerton and Punj, 1993). E-cigarettes are being used as devices to consume marijuana in a covert way as Sebastian explains:

...you're much less likely to be caught [smoking marijuana] if you were vaping. Because it's like you don't see like weed...you don't smell it as much because it's vaporized, it's not as much smoke. You don't see it as much because there literally isn't as much smoke. Like it's harder to tell. 
Using e-cigarettes for this purpose represents a clear shift in ritual boundaries particularly given it is marketed as a cessation aid for smoking nicotine (Cahn and Siegel, 2011). It is also more difficult to contest boundaries or manage consumer 'misbehavior' when practices are changed or altered. Concerns regarding the use of e-cigarettes as a gateway for youth to embark on tobacco consumption as a consequence of vaping are not realized in this study but clearly concerns regarding substance abuse in this context and the longer-term implications therein is worthy of further exploration.

Further anti-establishment type behavior is also evident amongst Vulnerable Vapers in the form of resistance (Cherrier, 2009) through vaping. This topic emerges in the participants' attitudes and behaviors towards established social norms of smoking behavior. Although legislation regulating where people can vape and whether vapers are subject to the same restrictions as tobacco smokers is mired in controversy (Vardavas et al., 2014), decades of anti-smoking campaigns alongside legislative change mean there are recognized boundaries where smoking in public places such as cinemas, restaurants and airplanes are deemed both unacceptable and prohibited by law. The result is a dominant cultural norm that causes smoking in such places to be unacceptable. However, Vulnerable Vapers engage in two types of active resistance to these norms by expressing dissatisfaction (Ward and Ostrom, 2006) with current legislation which treats vaping in the same way to tobacco smoking and through social displays of resistance (Maxwell, 2003) by vaping in smoke free environments. Jake and Sebastian describe their active resistance to smoke-free environments and illustrate the difficulties with implementing social policies when ritual boundaries shift:

'Cause honestly, you know, I don't think it's that big of a deal to [vape] inside of a movie theater or inside of a restaurant. (Jake)

I used to vaporize a lot on the fourth floor of the [University] Library. Um, you know like those late night papers, three or four in the morning. That was probably, maybe like the most inappropriate place that I used to vaporize. (Sebastian)

While, Anthony's experience illustrates that the University police consider vaping as equivalent to cigarette smoking, this policy may not reflect practices on other university campuses:

Now like I don't use [my e-cigarette] on campus. Even though I actually -- I looked up the smoking policy, and apparently there's no -- there's nothing against e-cigs on campus...but a cop stopped me and told me not to use it on campus like a year ago. 
And so, you know, I don't out of respect for them, and also not wanting to get stopped again, but it's annoying.

Comparatively with all other segments, Vulnerable Vapers exhibit the highest levels of resistance to social norms. Their behaviors are contrary to Virtuous Vapers who express conformity to current ritual boundaries that relate to smoke-free environments and illustrate their desire to vape in adherence to these established social norms. For Vulnerable Vapers, often the attractiveness of vaping is rooted in carousing, the sociability of this activity and its use as a form of resistance. In this way this segment can be identified as a group most at risk from the deconstruction and reassembling of e-cigarettes (Cova et al., 2007) and those illustrating most fluidity in terms of ritual boundaries. Less easy to identify are the Invisible Vapers as they are characteristically not socially engaged with other vapers.

\section{Invisible Vapers}

Unlike Dynamic Vapers, Invisible Vapers are the most reluctant to engage in social vaping practices comparatively with all other segments. This reluctance is apparent in their desire to disassociate with the vaping reference group (White and Dahl, 2006) which leads to more individualized vaping behavior. Broadly, this group actively rejects the social experience of vaping both in an attempt to manage the undesired self (Hogg and Banister, 2001; Ogilvie, 1987) and to protect self-esteem from what they perceive to be negative vaping stereotypes. While Invisible Vapers are motivated to vape primarily for the perceived health benefits they differ from Virtuous Vapers in their enthusiasm to learn about vaping or to evangelize their behavior in any way as Amy explains:

My boyfriend started vaping and, I don't know, the benefits just seemed better. As to smoking, well doing the vape rather than smoking, with the second hand smoke and the two kids...Um, I kind of just go off what my boyfriend tells me, because he is the expert.

Here Amy is conscious of the impact of her vaping behavior on others, in this case her children. Invisible Vapers also demonstrate a strong awareness of how their vaping behavior is perceived by others. For Jared this manifests in a desire to smoke individually and not in a group whilst Melissa wishes to "disidentify" (Englis and Solomon, 1995) with the vaping reference group. This behavior reflects negative or low EE whereby individuals are not attracted to the symbols of the group but rather feel a sense of alienation (Collins, 2004). 
I tend not to do it with like a lot of people around...just because I don't want to have that effect of people just assuming [I'm smoking tobacco] and judging [me]. (Jared) Honestly, when I see people vaping, I think they're annoying. I'm like, that's why I don't like doing it in public, because I don't want to be annoying. I think it's annoying because people are so like...I hate when people are like, this is what I do and I love it so much that you should do it too. You know, and that's how I think most of the vaping community is... (Melissa).

The group avoidance (White and Dahl, 2006) displayed by Melissa demonstrates a reluctance to be associated with other vapers because she believes publicly vaping illustrates self-satisfaction and consequently has negative connotations. This avoidance leads to Melissa actively managing her possible selves (Cross and Markus, 1994) as she shows disdain at the potential of becoming an 'annoying' vaper. Comparatively Jared feels that although he is vaping he is being characterized as a smoker which carries negative connotations and creates cognitive dissonance. This dissonance relates to self-image and product image congruency issues (Ericksen and Sirgy, 1992) where the positive connection between the self-concept and a consumption decision has been disrupted. In order to protect self-esteem consumers could avoid purchasing (Hogg and Banister, 2001) however, among Invisible Vapers we see positive market engagement but negative perceptions of product symbolism. This results in the vaping community being regarded as a dissociative reference group (Englis and Solomon, 1995; Turner, 1991) which is regarded as producing low EE by Invisible Vapers.

\section{Conclusions}

This study has identified youth practices and boundary shifts associated with the contemporary low intensity ritual of vaping. Despite a plethora of studies examining tobacco smoking as well as literature on the influences and motivations for youth to engage in such practices, previous research has been scant on vaping behaviors as well as the sociability it affords. As new products are introduced in the marketplace, behaviors can alter with changes to associated rituals increasingly difficult to oppose. Importantly, our findings offer a variety of vaping 'types' with related characteristics which both reflect and build on previous research that explores tobacco smokers and their behaviors. For example, our Vulnerable Vapers demonstrated that they could be led by the 'bandwagon effect' raising demand for vaping through peer interaction. This is consistent with studies on youth smoking behaviors (DeCicca et al., 2000; Liebenstein, 1950). Importantly, however, we find that Virtuous 
Vapers not only seek the health benefits offered by e-cigarettes but are advocates of this practice; pursuing information to reinforce vaping as a 'cleaner' alternative to smoking. The interpretation and meaning of vaping for Virtuous Vapers relative to Vulnerable Vapers is opposite with the former emphasizing the importance of conforming to social norms when vaping and the latter using vaping as a means of resistance and/or misbehavior.

Our findings also illustrate that contrary to concerns relating to vaping as a gateway for youth cigarette smoking, vapers who are sensation seeking (Màsse and Tremblay, 1997), rebellious (Burt, 2001), and more likely to engage in misbehavior or disorderly conduct (McMahon, 1999) are more inclined to use e-cigarettes as a covert means of illicit drug (marijuana) use. While "deviance prone" youth will be more likely to be involved in associated precarious behaviors (Chassin et al., 2007) identifying and supporting those prone to consumer misbehavior may mediate the longer-term risks for this group.

Our research contributes to extant studies in three ways. Firstly, through the lens of interaction rituals the findings illustrate that barriers to access differ for vaping and tobacco consumption in relation to customization, information seeking and knowledge transfer. Previous researchers note that smoking tobacco affords the opportunity to withdraw or escape, show defiance and facilitate an identity position (Domzal and Kernan, 1992; Hirschman and Holbrook, 1982). These behaviors are also observed here in the context of vaping. Our examination of this contemporary practice, however, shows that vapers are not a homogenous group and that their motivations for engaging in this practice vary considerably; with differences evident between those concerned with the health and functional benefits and individuals interested in the personalization, symbolism and social capital vaping can afford. Our research establishes the nuances and motives that lead to inconsistent practices associated with this low intensity vaping ritual. Commitment to vaping can also be viewed as practical or figurative. That is, vapers can invest in related vaping equipment to reduce associated costs. Alternatively, others are less concerned with price; benefitting from the way in which symbols facilitate self-identity positioning, social interaction and commonality or solidarity.

Secondly while we anticipated vaping would reflect tribal practice, this study revealed that while playfulness and creativity are characteristic of the Dynamic and Vulnerable Vapers, there is also evidence of opinion leadership as well as situational stratification which is more akin to sub-culture hierarchy (Cova and Pace, 2006). Importantly knowledge associated with this relatively new practice e.g. appropriate 'rigs', tricks, customization of devices and modifying liquids means accessing this practice typically necessitates more 
engagement than tobacco smoking. Our research extends previous findings by introducing an overlap between tribal and sub-cultural characteristics. While the dissemination of knowledge can lessen hierarchical boundaries (Lam, 2000) it can nevertheless create and/or amplify situational stratification.

Thirdly we identify a vaping segment that is resistant to the vaping 'community' but not to vaping per se. Invisible Vapers can be considered as strangers (Gronnow, 2004) to vaping groups or the vaping community. While the Vulnerable Vapers use e-cigarettes to challenge social norms, Invisible Vapers conform by replacing tobacco with e-cigarettes but are concerned with how they believe vaping is perceived by others. The marketplace engagement of Invisible Vapers appears contrary to the attitude-behavior gap and is worthy of further exploration. Interestingly, studies of organizational disidentification illustrate that individuals who disidentify with an organization tend to have limited personal experience with the organization. Consequently they hold stereotypical views about its members and possess narrow and simplified information regarding the organization (See Elsbach and Bhattacharya, 2001). The lack of engagement with other vapers may explain the (mis)perceptions of Invisible Vapers although this proposition would require further research.

The findings here also highlight a number of issues that can potentially be addressed or actioned through public policy; ensuring continuation and consistency of smoke-free environments, support and education for those using vape equipment to 'misbehave' and a harnessing of the advocacy of vaping as an alternative to tobacco smoking by Virtuous Vapers.

\section{Further research}

As the current study was exploratory in nature and as such utilized a relatively small sample of vapers, future research could use quantitative techniques on larger samples of those consuming e-cigarettes to offer more generalizable insights on how and why they engage in such practices and the relative outcomes. A future study could also examine the role of multiple selves to further understand the role of identity and vaping. For example, do vapers change their vaping 'self' dependent on the (social) context or environment in which they are vaping? Furthermore, the evolving legislation around vaping means vape shops and cafes are emerging as hubs for collective vaping participation. A future study could take an ethnographic approach to examine these locations to better understand their role in vaping culture. Given that vaping has been established as a heterogeneous market where individual motivations for engagement vary, it would also be interesting to understand what impact the 
rising normalization of vaping is having on current tobacco smokers, non-smokers and vapers themselves. Similarly the way in which everyday sociability is impacted through vaping practices and the inter-relationships between and amongst tobacco smokers and vapers is worthy of further exploration. Finally, the Invisible Vapers identified in this study hold particularly strong views about how others perceive their vaping behavior. Future research could explore the perception of others in more detail considering the different opinions held within the vaping community of each other and then how others characterize the vaping community. 


\section{References}

Albritton K. 2015. Milennial misunderstanding? Capstrat. Available at https://www.capstrat.com/elements/downloads/files/millennial-whitepaper.pdf [accessed on 3 January 2015].

Arnett, Jeffrey Jensen. 2007. Emerging adulthood: What is it, and what is it good for?. Child development perspectives $1(2)$ 68-73.

Belk RW, Wallendorf M, Sherry JF. 1989. The sacred and the profane in consumer behavior: theodicy on the Odyssey. Journal of Consumer Research 16(1): 1-38.

Bialous S, Sarma L. 2013. E-cigarettes: a moral quandary. Lancet 382(9896): 914.

Blau PM, Scott RW. 1962. Formal Organizations. Chandler: San Francisco, CA.

Bonsu S. 2001. Death and identity: consumer behavior in Asante death rituals. Dissertations and Master's theses paper AAI3025531. Available at http://digitalcommons.uri.edu/dissertations/AAI3025531 [accessed on 15 December 2015].

Borland R. 2011. Electronic cigarettes as a method of tobacco control. BMJ 343: d6269.

Bourdieu P. 1986. The forms of capital, in Handbook of Theory and Research for the Sociology of Education, John G Richardson (ed.). Greenwood Publishing Group: New York.

Brown J, Beard E, Kotz D, Michie S, West R. 2014. Real-world effectiveness of e-cigarettes when used to aid smoking cessation: a cross-sectional population study. Addiction 109(9): 1531-1540.

Brown KR. 2011. Interaction ritual chains and the mobilization of conscientious consumers. Qualitative Sociology 34(1): 121-141.

Brown S. 2007. Harry Potter and the fandom menace, in Consumer Tribes, Bernard Cova, Robert Kozinets, Avi Shankar (eds.). Butterworth-Heinemann: Amsterdam.

Brownlie D, Hewer P, Treanor S. 2007. Sociality in motion: exploring logics of tribal consumption, in Consumer Tribes, Bernard Cova, Robert Kozinets, Avi Shankar (eds.). Butterworth-Heinemann: Amsterdam.

Burt RS. 2001. Structural holes versus network closure as social capital, in Social Capital: Theory and Research, Nan Lin, Karen Cook, Ronald Burt (eds.). Aldine Transaction: New Brunswick, NJ.

Cahn Z, Siegel M. 2011. Electronic cigarettes as a harm reduction strategy for tobacco control: a step forward or a repeat of past mistakes? Journal of Public Health Policy 32(1): 16-31. 
Calhoun CCH. 1980. The human moral sentiment: a unique feeling. Southwestern Journal of Philosophy 11(1): 69-78.

Centers for Disease Control and Prevention. 2013. About one in five US adult cigarette smokers have tried an electronic cigarette. Available at http://www.cdc.gov/media/releases/2013/p0228_electronic_cigarettes [accessed on 3 January 2015].

Centers for Disease Control and Prevention. 2015. E-cigarette use triples among middle and high school students in just one year. Available at http://www.cdc.gov/media/releases/2015/p0416-e-cigarette-use.html [accessed on 3 January 2015].

Chassin, L., Pillow, D.R., Curran, P.J., Molina, B.S. and Barrera Jr, M., 1993. Relation of parental alcoholism to early adolescent substance use: a test of three mediating mechanisms. Journal of abnormal psychology, 102(1), p.3.

Chassin L, Presson C, Morgan-Lopez A, Sherman SJ. 2007. Deviance proneness' and adolescent smoking 1980 versus 2001: has there been a hardening of adolescent smoking? Journal of Applied Developmental Psychology 28(3): 264-276.

Cherrier H. 2009. Anti-consumption discourses and consumer-resistant identities. Journal of Business Research 62(2): 181-190.

Choi K, Forster J. 2013. Characteristics associated with awareness, perceptions, and use of electronic nicotine delivery systems among young US midwestern adults. American Journal of Public Health 103(3): 556-561.

Collins R. 2004. Interaction Ritual Chains. Princeton University Press: Princeton.

Cova B, Cova V. 2002. Tribal marketing. European Journal of Marketing 36(5/6): 595-620.

Cova B, Kozinets RV, Shankar A. 2007. Consumer Tribes. Butterworth-Heinemann: Amsterdam.

Cova B, Pace S. 2006. Brand community of convenience products: new forms of customer empowerment - the case "my Nutella the community". European Journal of Marketing 40(9/10): 1087-1105.

Cross SE, Markus HR. 1994. Self-schemas, possible selves, and competent performance. Journal of Educational Psychology 86(3): 423-438.

DeCicca P, Kenkel D, Mathios A. 2000. Racial difference in the determinants of smoking onset. Journal of Risk \& Uncertainty 21(2/3): 311-340.

Deighton J. 1992. The consumption of performance. Journal of Consumer Research 19(3): 362-372. 
Domzal TJ, Kernan JB. 1992. Reading advertising: the what and how of product meaning. Journal of Consumer Marketing 9(3): 48-64.

Durkheim E. 1954. The Elementary Forms of Religious Life. Free Press: New York.

Durkheim E. 1964. The Division of Labor in Society. Free Press: New York.

Elsbach KD, Bhattacharya CB. 2001. Defining who you are by what you're not: organizational disidentification and the National Rifle Association. Organization Science 12(4): 393-413.

Englis BG, Solomon MR. 1995. To be and not to be: lifestyle imagery, reference groups, and the clustering of America. Journal of Advertising 24(1): 13-28.

Ericksen MK, Sirgy MJ. 1992. Employed females' clothing preference, self-image congruence, and career anchorage. Journal of Applied Social Psychology 22(5): 408422.

Etter J.-F. 2010. Electronic cigarettes: a survey of users. BMC Public Health 10: 231.

Etter J.-F, Bullen C. 2011. Electronic cigarette: users profile, utilization, satisfaction and perceived efficacy. Addiction 106(11): 2017-2028.

Euromonitor. 2012. E-cigarettes: a US\$ 2 billion global industry - who should be worried? Available at http://blog.euromonitor.com/2012/11/e-cigarettes-a-aus2-billion-globalindustry-who-should-be-worried.html [accessed on 17 September 2013].

Fullerton RA, Punj G. 1993. Choosing to misbehave: a structural model of aberrant consumer behavior. Advances in Consumer Research 20: 570-574.

Gerrard, M., Gibbons, F.X., Benthin, A.C. and Hessling, R.M., 1996. A longitudinal study of the reciprocal nature of risk behaviors and cognitions in adolescents: what you do shapes what you think, and vice versa. Health psychology, 15(5), p.344.

Gilbert, D.G., 1995. Smoking: individual difference, psychopathology, and emotion. Taylor \& Francis.

Gilman S, Zhou X. 2004. Smoke. Reaktion Books: London.

Goffman E. 1967. Interaction Rituals: Essays in Face to Face Behavior. Anchor Books: New York.

Goulding C, Saren M. 2007. Gothic, entrepreneurs: a study of the subcultural commodification process, in Consumer Tribes, Bernard Cova, Robert Kozinets, Avi Shankar (eds.). Butterworth-Heinemann: Amsterdam.

Goulding C, Shankar A, Canniford R. 2013. Learning to be tribal: facilitating the formation of consumer tribes. European Journal of Marketing 47(5/6): 813-832. 
Goulding C, Shankar A, Elliott R. 2002. Working weeks, rave weekends: identity fragmentation and the emergence of new communities. Consumption Markets \& Culture 5(4): 261-284.

Gronnow J. 2004. Standards of taste and varieties of goodness: the (un)predictability of modern consumption, in Theoretical Approaches of Food Quality, Mark Harvey, Andrew McMeekin, Alan Warde (eds.). Manchester University Press: Manchester; $38-60$.

Guest G, Bunce A, Johnson L. 2006. How many interviews are enough?: an experiment with data saturation and variability. Field Methods 18(1): 59-82.

Hampson, S.E., Andrews, J.A. and Barckley, M., 2007. Predictors of the development of elementary-school children's intentions to smoke cigarettes: Hostility, prototypes, and subjective norms. Nicotine \& Tobacco Research, 9(7), pp.751-760.

Hendlin Y, Anderson SJ, Glantz SA. 2010. Acceptable rebellion: marketing hipster aesthetics to sell Camel cigarettes in the US. Tobacco Control 19(3): 213-222.

Hirschman EC, Holbrook MB. 1982. Hedonic consumption: emerging concepts, methods and propositions. Journal of Marketing 46(3): 92-101.

Hogg MK, Banister EN. 2001. Dislikes, distastes and the undesired self: conceptualising and exploring the role of the undesired end state in consumer experience. Journal of Marketing Management 17(1-2): 73-104.

Hughes-Freeland F, Crain MM. 1998. Recasting Ritual: Performance, Media, Identity. Routledge: London.

Kemper TD. 1990. Research Agendas in the Sociology of Emotions. SUNY Press: Albany.

Lam A. 2000. Tacit knowledge, organizational learning and societal institutions: an integrated framework. Organization Studies 21(3): 487-513.

Leventhal H, Keeshan P, Baker T, Wetter D. 1991. Smoking prevention: towards a process approach. British Journal of Addiction 86(5): 583-587.

Liebenstein H. 1950. Bandwagon, snob, and Veblen effects in the theory of consumer demand. Quarterly Journal of Economics 64: 183-207.

Marlow M. 2014. Regulating a less unhealthy cigarette. Heartland Institute 28.

Màsse LC, Tremblay RE. 1997. Behavior of boys in kindergarten and the onset of substance use during adolescence. Archives of General Psychiatry 54(1): 62-68.

Maxwell I. 2003. Phat Beats, Dope Rhymes: Hip Hop Down Under Comin' Up. Wesleyan University Press: Middletown. 
McAlexander J. 1991. Divorce, the disposition of the relationship, and everything. Association for Consumer Research 18: 43-48.

McCracken G. 1986. Culture and consumption: a theoretical account of the structure and movement of the cultural meaning of consumer goods. Journal of Consumer Research 13(1): 71-84.

McMahon RJ. 1999. Parent training in handbook of psychotherapies with children and families, S Russ, T Ollendick (eds.). Kluwer Academic/Plenum: New York; 153-180.

Miles M, Huberman M. 1994. Qualitative Data Analysis. Sage Publications: Thousand Oaks.

Mills PK, Margulies N. 1980. Toward a core typology of service organizations. Academy of Management Review 5(2): 255-266.

O'Callaghan F, Doyle J. 2001. What is the role of impression management in adolescent cigarette smoking? Journal of Substance Abuse 13(4): 459-470.

Ogilvie DM. 1987. The undesired self: a neglected variable in personality research. Journal of Personality \& Social Psychology 52(2): 379-385.

Otnes C, Lowrey TM. 1993. Til debt do US part: the selection and meaning of artifacts in the American wedding. Advances in Consumer Research 20(1): 325-329.

Ozanne J. 1992. The role of consumption and disposition during classic rites of passage: the journey of birth, initiation, and death. Advances in Consumer Research 19: 396-403.

Paradise J. 2014. Electronic cigarettes: smoke-free laws, sale restrictions, and the public health. American Journal of Public Health 104(6): e17-e18.

Rojek C. 2000. Leisure and the rich today: Veblen's thesis after a century. Leisure Studies 19(1): 1-15.

Rook, Dennis W. 1985. The ritual dimension of consumer behavior. Journal of Consumer Research: 251-264.

Room, R., 2004. Smoking and drinking as complementary behaviours. Biomedicine \& Pharmacotherapy, 58(2), pp.111-115.

Schlenker BR. 1980. The Self and Social Life. McGraw-Hill: New York.

Sheth JN, Newman BI, Gross BL. 1991. Why we buy what we buy: a theory of consumption values. Journal of Business Research 22(2): 159-170.

Shiu E, Hassan LM, Walsh G. 2009. Demarketing tobacco through governmental policies the 4Ps revisited. Journal of Business Research 62(2): 269-278.

Simmel G, Hughes EC. 1949. The sociology of sociability. American Journal of Sociology 55(3): 254-261.

Slater D. 1997. Consumer Culture and Modernity. Polity Press: Cambridge. 
Spiggle S. 1994. Analysis and interpretation of qualitative data in consumer research. Journal of Consumer Research 21(3): 491-503.

Strauss A, Corbin J. 1990. Basics of Qualitative Research: Grounded Theory. Sage Publishing House: Newbury Park.

Thompson CJ, Hirschman EC. 1995. Understanding the socialized body: a poststructuralist analysis of consumers' self-conceptions, body images, and self-care practices. Journal of Consumer Research 22(2): 139-153.

Turner JC. 1991. Social Influence. Open University Press: Buckingham.

Vardavas CI, Filippidis FT, Agaku IT. 2015. Determinants and prevalence of e-cigarette use throughout the European Union: a secondary analysis of 26566 youth and adults from 27 countries. Tobacco Control 24(5): 442-448.

Ward JC, Ostrom AL. 2006. Complaining to the masses: the role of protest framing in customer-created complaint web sites. Journal of Consumer Research 33(2): 220-230.

Wearing S, Wearing B. 2000. Smoking as a fashion accessory in the 90s: conspicuous consumption, identity and adolescent women's leisure choices. Leisure Studies 19(1): 45-58.

West, R.J., Jarvis, M.J., Russell, M.A.H., Carruthers, M.E. and Feyerabend, C., 1984. Effect of nicotine replacement on the cigarette withdrawal syndrome. British journal of addiction, 79(4), pp.215-219.

White K, Dahl DW. 2006. To be or not be? The influence of dissociative reference groups on consumer preferences. Journal of Consumer Psychology 16(4): 404-414. 
Table 1. Participant profile

\begin{tabular}{|c|c|c|c|c|c|c|}
\hline Interviewee pseudonym & Age & Gender & Occupation & Typology & Interviewed by a vaper or non-vaper & Former tobacco smoker \\
\hline Nate & 24 & M & gym instructor & Virtuous & Non-vaper & Yes \\
\hline Andy & 21 & M & student & Virtuous & Vaper & Yes \\
\hline Anthony & 22 & M & student & Virtuous & Vaper & Yes \\
\hline Ivan & 21 & M & entrepreneur & Virtuous & Vaper & No (occasionally cigars in the past but never cigarettes) \\
\hline Randy & 22 & M & finance administration & Virtuous & Vaper & Yes \\
\hline Deshaun & 22 & $\mathrm{~F}$ & student & Virtuous & Non-vaper & Yes \\
\hline Brad & 22 & M & vape store employee & Dynamic & Non-vaper & Yes \\
\hline Katie & 21 & $\mathrm{~F}$ & finance intern & Dynamic & Vaper & No \\
\hline Mike & 21 & M & waiter & Dynamic & Vaper & No (used tobacco to smoke marijuana but not cigarettes) \\
\hline Noel & 21 & $\mathrm{~F}$ & student & Dynamic & Vaper & Yes \\
\hline Duane & 21 & M & marketer & Dynamic & Non-vaper & No (used tobacco to smoke marijuana but not cigarettes) \\
\hline Amelia & 21 & $\mathrm{~F}$ & student & Vulnerable & Non-vaper & No \\
\hline Jake & 22 & M & student & Vulnerable & Non-vaper & Yes \\
\hline Tyrone & 22 & M & student & Vulnerable & Non-vaper & Yes \\
\hline Sebastian & 22 & M & student & Vulnerable & Vaper & No \\
\hline Alexa & 23 & $\mathrm{~F}$ & student & Vulnerable & Vaper & Yes \\
\hline Melissa & 21 & $\mathrm{~F}$ & student & Invisible & Non-vaper & Yes \\
\hline Jared & 24 & M & DJ (music) & Invisible & Non-vaper & Yes \\
\hline Amy & 27 & $\mathrm{~F}$ & stay-at-home-mom & Invisible & Non-vaper & Yes \\
\hline
\end{tabular}


Appendix 1. Interview questions (italics represent interviewer prompts)

\section{About your leisure time}

- How do you like to spend your leisure time?

Trying to get a sense of the person, who they are, how the spend their time, are they more sociable or loner types? What activities do they do? How big a part of their social life is vaping?

\section{About you}

- What does vaping say about you?

Identity. Do they tell others they vape? Do they vape in public where non-vapers are?

-What do you think about vaping compared to tobacco smoking?

Resistance to 'big tobacco'. Do they see the tobacco industry as 'the enemy'?

-What attracted you to vaping initially?

Means of escape, to relax, for fun

- Do you think vaping is kind of cool? If so why?

About your friends

Cool is all about rebellion, is vaping a rebellious statement?

- How important is it that you socialize with friends?

-What do your friends and family think about you vaping?

\section{Identity}

- Do you have different groups of friends - those who vape and those who don't?

\section{Vaping (general)}

- How often do you vape and where?

Really want to understand how they use vaping in their lives

Do they vape anywhere they 'technically' shouldn't be?

- How do you feel when you're with other vapers?

- Do you do anything different when you're vaping with friends rather than alone?

Playfulness, do they compare liquids, vaporizers, blow smoke clouds?

- How much do you think vaping is a creative or playful experience?

- Do you improvise in any way where you (and your friends) vape?

- Are you part of a vape group either physical or online?

How did they find this group?

How did they get to know people?

How did they feel when they first went along/took part compared to now?

- Are you part of more than one social group of vapers?

If so what are the differences? Are there collective values that distinguish the groups?

- How does it make you after vaping with a group of friends and you go home?

Is there emotional energy generated by being in the group?

e.g feel confident, proud, positive etc.?

\section{Vaping (knowledge)}

- Tell me about your first experience of vaping

Was it alone, with others?

- Tell me about your first experience of vaping with others

- How did you feel going in to a vape shop to buy things for the first time?

- How did you build up your knowledge about vaping techniques?

- Who do you trust to give you advice about vaping?

Any people/opinion leaders, particular websites etc. If so why those ones?

- Do you have specific vape shops that you go to or brands that you support?

- Or any that you avoid?

- Tell me about your vaporizer, is it the same one you always had?

There is a progression of simple to more complex vaporizers, what was driving the desire to get a new one? Group effect? Seen a friend with one etc. 


\section{Vaping (industry)}

- How do you feel about restrictions on being able to vape?

How do they get around this?

-What do you think about the idea that vaping is a gateway to smoking tobacco?

- Do you think vaping should be promoted as a way of helping people give up cigarette smoking? 


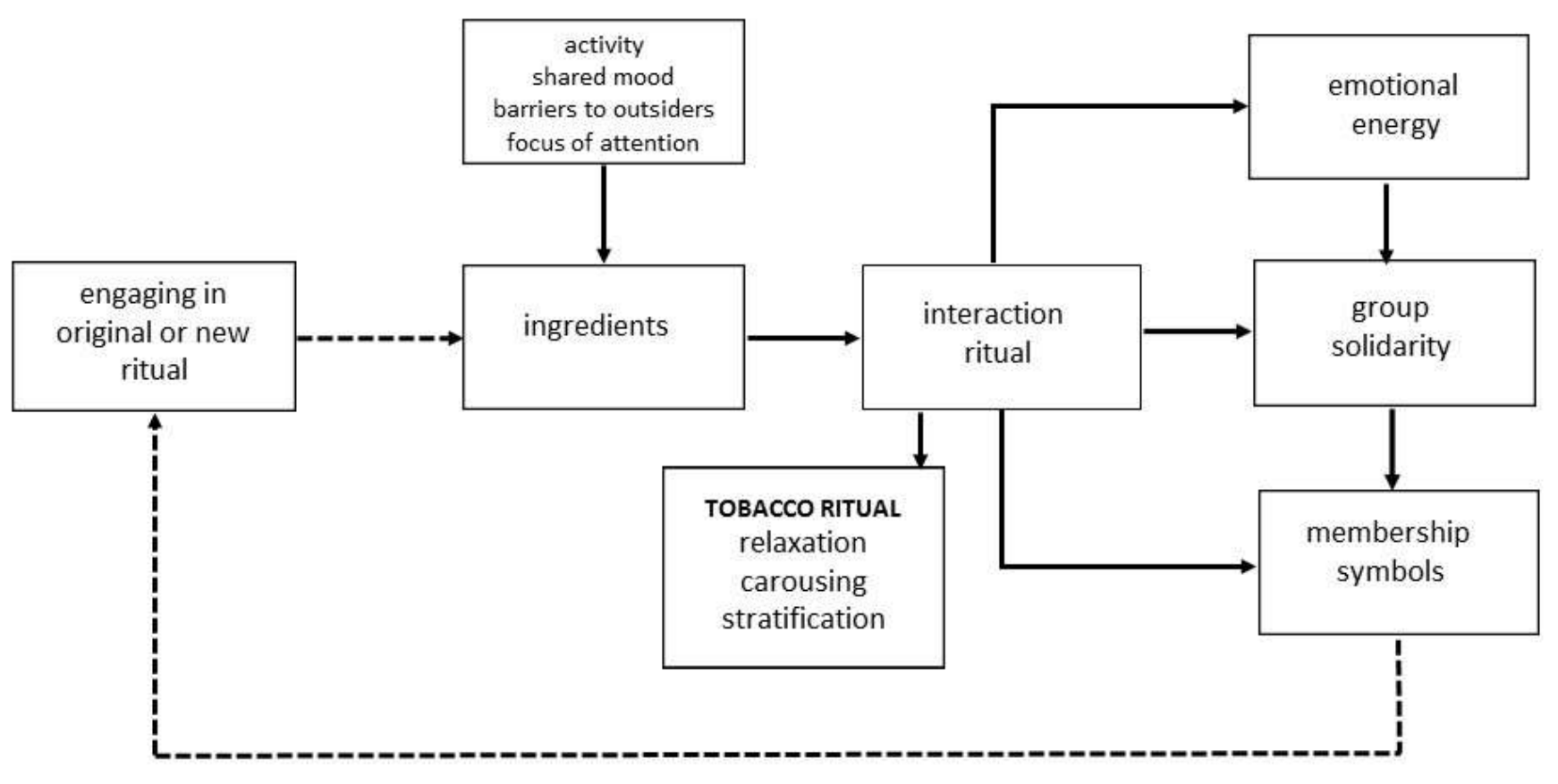

Figure 1. Interaction Ritual Chains (Adapted Collins, 2004). 
Importance of social experience

\section{LOW}

\section{Virtuous Vapers}

Ex-smokers

Interested in the mechanics of vaping

Focus on practicalities of use

Knowledge of playful/creative practices is

limited

Enjoy cost benefits

Enjoy convenience
HIGH

\section{Dynamic Vapers}

Exhibit playful behavior

Rarely vape individually

Generate most positive EE relative to

other groups

Demonstrate opinion leadership

Knowledge of creative practices is

extensive 


$\begin{array}{ll}\text { Invisible Vapers } & \text { Vulnerable Vapers } \\ \text { Vape individually } & \text { Only vapes socially } \\ \text { Peripheral members of vaping community } & \text { Exhibit most evidence of carousing } \\ \text { Follow without engaging } & \text { 'Misbehave' with vaping equipment } \\ \text { Experience most negative EE relative to } & \text { Push ritual boundaries } \\ \text { other groups } & \text { Tend towards anti-establishment and } \\ \text { Social group members often smokers } & \text { 'resist' through vaping }\end{array}$

Figure 2. Vaping Typologies. 